\title{
Higher breakfast glycaemic load is associated with increased metabolic syndrome risk, including lower HDL-cholesterol concentrations and increased TAG concentrations, in adolescent girls
}

\author{
Analise Nicholl ${ }^{1} \dagger$, Mary du Heaume ${ }^{1} \dagger$, Trevor A. Mori ${ }^{2}$, Lawrence J. Beilin ${ }^{2}$, Wendy H. Oddy ${ }^{3}$, \\ Alexandra P. Bremner ${ }^{4}$ and Therese A. O'Sullivan ${ }^{1 *}$ \\ ${ }^{1}$ School of Exercise and Health Science, Edith Cowan University, 270 Joondalup Drive, Joondalup, WA, Australia \\ ${ }^{2}$ School of Medicine and Pharmacology, University of Western Australia, Perth, WA, Australia \\ ${ }^{3}$ Telethon Kids Institute, University of Western Australia, West Perth, WA, Australia \\ ${ }^{4}$ School of Population Health, University of Western Australia, Perth, WA, Australia \\ (Submitted 20 January 2014 - Final revision received 14 August 2014 - Accepted 26 August 2014 - First published online 20 October 2014)
}

\section{Abstract}

Almost all previous studies examining the associations between glycaemic load (GL) and metabolic syndrome risk have used a daily GL value. The daily value does not distinguish between peaks of GL intake over the day, which may be more closely associated with the risk of the metabolic syndrome. The aim of the present study was to investigate the cross-sectional associations between daily and mealtime measures of GL and metabolic syndrome risk, including metabolic syndrome components, in adolescents. Adolescents participating in the 14-year follow-up of the Western Australian Pregnancy Cohort (Raine) Study completed $3 \mathrm{~d}$ food records and metabolic assessments. Breakfast GL, lunch GL, dinner GL and a score representing meal GL peaks over the day were determined in 516 adolescents. Logistic regression models were used to investigate whether GL variables were independent predictors of the metabolic syndrome in this population-based cohort (3.5\% prevalence of the metabolic syndrome). Breakfast GL was found to be predictive of the metabolic syndrome in girls (OR 1.15, $95 \%$ CI 1.04, 1.27; P<0.01), but not in boys. Other meal GL values and daily GL were found to be not significant predictors of the metabolic syndrome. When breakfast GL was examined in relation to each of the components of the metabolic syndrome in girls, it was found to be negatively associated with fasting HDL-cholesterol concentrations $(P=0 \cdot 037 ; \beta=-0 \cdot 004 ; 95 \% \mathrm{CI}-0 \cdot 008$, $-0.002)$ and positively associated with fasting TAG concentrations $(P=0.008$; $\exp (\beta)=1.002 ; 95 \%$ CI $1.001,1 \cdot 004)$. The results of the present study suggest that there may be an association between breakfast composition and metabolic syndrome components in adolescent girls. These findings support further investigation into including lower-GL foods as part of a healthy breakfast in adolescence, particularly for girls.

Key words: Metabolic syndrome: Glycaemic load: Adolescents: Breakfast: Raine Study

The metabolic syndrome is a cluster of metabolic disturbances that increases the risk of developing type 2 diabetes and $\mathrm{CVD}^{(1,2)}$. In Australia, the prevalence of the metabolic syndrome in adolescents has previously been reported to be $3.6 \%$ using the paediatric diagnostic criteria of the International Diabetes Federation (IDF) ${ }^{(3)}$, increasing to $22 \cdot 1 \%$ in adulthood (adult IDF criteria) ${ }^{(4)}$. Diet is one of the factors that may have the ability to influence this progression from adolescence to adulthood.

The glycaemic index (GI) was proposed over 30 years ago with the aim of improving postprandial glycaemia in the diabetic population $^{(5)}$. The GI ranks foods or beverages based on their ability to raise blood glucose concentrations compared with the ingestion of the same quantity of carbohydrate, expressed as a percentage. A high-GI food consumed in a small amount can have a minimal impact on blood glucose concentrations and conversely a low-GI food consumed in a large amount can have a major impact on blood glucose concentrations. The glycaemic load (GL) is a product of the quantity of carbohydrate present in the food and the GI; by taking the carbohydrate into consideration, it represents the total impact of the food on blood glucose concentrations ${ }^{(6)}$. Hence, the GL is better able to distinguish the impact on postprandial glycaemia compared with the GI.

Abbreviations: GI, glycaemic index; GL, glycaemic load; HDL-C, HDL-cholesterol; IDF, International Diabetes Federation.

*Corresponding author: T. O'Sullivan, fax +61 863045384 , email t.osullivan@ecu.edu.au

† Both authors contributed equally to the present study. 
Habitual intake of a high-GL diet may lead to hyperinsulinaemia and disturbed lipid metabolism ${ }^{(7)}$, with an increased risk of developing the metabolic syndrome ${ }^{(8,9)}$. Lower-GI/GL diets have been reported to be associated with improved health outcomes for various metabolic risk factors and chronic diseases in studies and meta-analyses ${ }^{(10-12)}$. Some studies have found no significant association between low-GI/GL diets and reduced risk of diabetes ${ }^{(13,14)}$, perhaps in part because the use of daily values has some limitations in representing metabolic processes resulting from habitual dietary carbohydrate intake over the course of the day ${ }^{(13)}$. Studies investigating associations with dietary GI and GL often use FFQ, which can estimate daily GI/GL, but not individual meal values. We identified two studies that were able to assess meal values using either a food record (Hong Kong children aged 6-7 years ${ }^{(15)}$ ) or diet history (older Australian women $\left.{ }^{(16)}\right)$. The latter considered a new measure of high glycaemic carbohydrate impact, the GL peak score, based on the summation of individual mealtime GL values that scored a peak above the daily GL mean ${ }^{(16)}$. To date, no published adolescent studies appear to have examined the mealtime patterns of glycaemic impact, including investigation of periods when GL intake may peak substantially.

The determination of carbohydrate intake patterns may provide insights into potential glycaemic impacts for adolescents, who are also exposed to growth stresses and metabolic and hormonal changes. The aim of the present explorative study was to investigate the mealtime measures of GL intake in relation to metabolic syndrome risk, as well as components of the metabolic syndrome, during the 14-year follow-up of the Western Australian Pregnancy Cohort (Raine) Study in Perth, Western Australia. We hypothesised that individual meal GL values and a score representing peaks in meal GL would be better predictors of metabolic syndrome risk than a daily GL value.

\section{Research design and methods}

\section{Study population}

The present study is a cross-sectional analysis of adolescents who participated in the 14-year follow-up of the Raine Study. As has been described previously ${ }^{(17)}$, 2900 pregnant women were enrolled in a controlled trial from public and private antenatal clinics at or near King Edward Memorial Hospital in Perth, Western Australia, between May 1989 and November 1991. The resulting 2868 children were recruited for cohort follow-up. The 14-year follow-up (mean age $14 \cdot 0$ (SD 0.2 ) years; age range $13.0-15.0$ years) occurred from 2003 to 2005 and was the first to collect comprehensive dietary intake data allowing nutrient analysis of individual meals in a habitual diet. Adolescents with type 1 or type 2 diabetes mellitus or implausible energy intakes $(<3000$ or $>20000 \mathrm{~kJ} / \mathrm{d}$, as has been used previously in studies involving adolescents $\left.{ }^{(18,19)}\right)$ were excluded from the study. Written informed consent for performing the 14-year followup procedures was provided by study participants and a parent/guardian, and approval was obtained from the ethics committees of King Edward Memorial Hospital and Princess Margaret Hospital for Children.

\section{Dietary glycaemic intake assessment}

The adolescents completed $3 \mathrm{~d}$ food records, with parental support if requested. Intakes were recorded in household measures. The subjects were provided with written and verbal instructions, as well as metric measuring cups and spoons. Consumption away from home was recorded in relation to serving size (e.g. two slices of a large pizza or one Whopper hamburger) or estimated in household measures. A checklist was used to ascertain whether each of the $3 \mathrm{~d}$ records was typical of the subject's usual intake, and only those records completed and classified as representative were used. A dietitian checked each food record as it was returned and sought clarification via follow-up telephone calls $^{(20)}$. Food record data were entered into FoodWorks dietary analysis software (Professional version 4.00; Xyris Software). Food composition data that were not available through FoodWorks were obtained from an Australian nutrition website with a customised GI database ${ }^{(21)}$. Where GI values for a specific product were not available, the GI value was imputed from a product or subgroup of products that was assessed by the researchers to be sufficiently similar in terms of type of starch, molecular monosaccharide components, ingredients, including amounts of protein and fat and amount of dietary fibre present, and degree of cooking or processing. If a product was too specialised to be a good match (e.g. a specific type of body-building powder), no GI value was assigned. GI values for mixed foods and recipes were estimated from component foods; for example, the GI for trifle was based on a weighted GI calculation of the carbohydrate-containing ingredients (sponge, jelly and custard). The formula used to calculate the composite GI of meals based on relative weighting of carbohydrate content does not take into account the effect of the whole dish, and there is likely to be a variable loss of discrimination of individual GI values in composite foods.

To ensure that food records were representative, only food records with $80 \%$ or more of the total daily dietary carbohydrate assigned a GI value were included in this study. GL values for individual meals comprised the sum of GL values for all foods and beverages in that meal. Meal GL values were obtained by averaging the values for each particular meal over the $3 \mathrm{~d}$ record period to obtain daily breakfast, morning tea, lunch, afternoon tea, dinner and supper GL values for each subject. Limited availability of GI values may affect the results of studies examining associations between GI/GL and chronic diseases, particularly when local/traditional foods are involved. For the present cohort, it was possible to assign GI values to $92 \%$ of all carbohydrate foods and beverages ${ }^{(3)}$. This meant that for some subjects carbohydrate foods or beverages in a meal could not be assigned a GI value. Non-assignment of a GI value meant that the contribution of these foods or beverages to the meal GL could not be calculated (despite having a likely effect on blood glucose concentrations). To ensure that the meal GL 
values that were being used were as representative as possible of the food being consumed, it was decided that $80 \%$ or more of the dietary carbohydrate per meal should be assigned a GI value in order for the meal GL to be used in the study (consistent with the daily criterion). This was based on methods used in previous research and professional opinion of clinical relevance, whereby a value lower than $80 \%$ was thought to potentially compromise the validity of the data ${ }^{(3)}$. Subjects were excluded if this meant that two or more meals of the same type (e.g. breakfast) over the $3 \mathrm{~d}$ record period did not have usable GL values.

Mean breakfast GL, morning tea GL, lunch GL, afternoon tea GL, dinner GL and supper GL were calculated for each subject where possible. Together with the mean meal GL (the mean of the above six meal GL values), these were used to obtain the peak GL score. Meal peak GL values were calculated for each subject by subtracting the mean meal GL value from the GL value of each meal and represented graphically as a set of positive and negative peaks with the mean set to zero. The peak GL score was calculated by adding all the positive meal peak values $^{(16)}$ (Fig. 1). For the purposes of the present study, five GL variables were investigated: breakfast GL; lunch GL; dinner GL; peak score GL; daily GL.

\section{Metabolic syndrome definition}

The prevalence of the metabolic syndrome in the present adolescent cohort at the 14-year follow-up has previously been reported to be 3.6 or $4.0 \%{ }^{(3)}$, using age-specific adolescent definitions of the IDF and the National Cholesterol Education Program Adult Treatment Panel III, respectively ${ }^{(22)}$. Although no consistent adolescent definition for the metabolic syndrome exists, the American Heart Association recommends using the IDF paediatric definition for adolescents ${ }^{(23)}$, and this was used in the present study. The IDF metabolic syndrome definition requires the presence of a high waist circumference in addition to two or more of the following: high systolic or diastolic blood pressure; high fasting serum TAG concentrations; low fasting serum HDL-cholesterol (HDL-C) concentrations; high fasting plasma glucose concentrations. Cut points for the categorisation of these high and low subgroups vary by sex and age, as has been published previously ${ }^{(22)}$. A research nurse took waist measurements at the level of the umbilicus in adolescents standing in the anatomical position to the nearest $0 \cdot 1 \mathrm{~cm}$ until two readings were within a $\mathrm{cm}$ of each other. Phlebotomists visited adolescents at their homes to obtain fasting blood samples. Serum glucose concentrations were measured using an automated Technicon Axon analyser (Bayer Diagnostics); TAG concentrations were measured using the Cobas MIRA Analyser (Roche Diagnostics); and HDL-C concentrations were measured on a heparin-Mn supernatant. Biochemical assays were conducted by PathWest Laboratories at Royal Perth Hospital. At rest over a $10 \mathrm{~min}$ period, six measurements of seated blood pressure readings were taken using a Dinamap ProCare 100 automatic oscillometric recorder (GE Healthcare Technologies). The first measurement was disregarded, and the mean of the next five measurements was calculated to obtain diastolic and systolic blood pressure values.

\section{Potential confounding variables}

Information regarding potential confounding variables was collected from adolescents themselves and their parents/ guardians ${ }^{(3)}$. Physical and sedentary activity levels were assessed by time spent outside school hours participating in physical activity that caused breathlessness or sweating (categorised as less than once a week $=$ low exercise, one to three times a week $=$ moderate exercise, or four or more times a

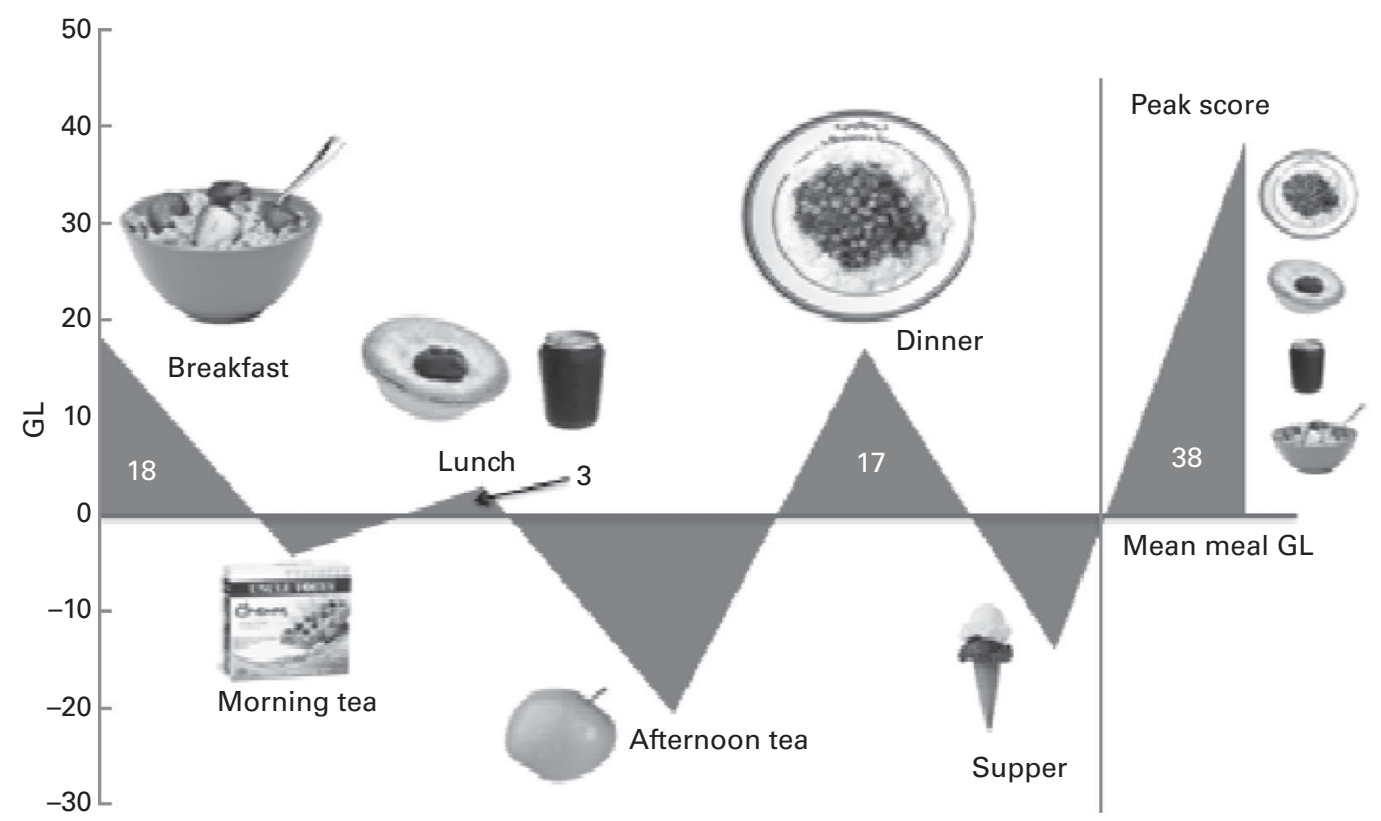

Fig. 1. Glycaemic load (GL) variables and food intake for a sample subject in the Raine Study (chosen for illustrative purposes only). The mean meal GL was set to zero, producing both positive and negative peaks. For this subject, positive peaks were observed at breakfast (18), lunch (3) and dinner (17). These were summed to obtain the peak GL score, which was 38 (sum of positive peaks). 
week = high exercise) and time spent watching television/ videos and using computers for school, work and recreation (categorised as $<2 \mathrm{~h} / \mathrm{d}=$ low screen use, $2-4 \mathrm{~h} / \mathrm{d}=$ moderate screen use, or $>4 \mathrm{~h} / \mathrm{d}=$ high screen use). These variables were combined into a five-category summary variable, which ranged from low screen use with more exercise to high screen use with less exercise. Details regarding family characteristics including family structure, family income, maternal age, maternal education and family history of diabetes and CVD were obtained from parental reports. The Tanner stages of pubic hair development were used to assess pubertal status in the cohort ${ }^{(24,25)}$. Adolescents were asked to select their corresponding developmental stage from a set of standard drawings depicting Tanner stages 2 (sparse) to 5 (adult) in a privately completed questionnaire. Stage 1 was omitted as an option as it corresponds to a pre-pubescent period ( $<10$ years of age). Dietary variables considered as potential confounding factors in the models included average daily intakes of total energy, total fat, saturated fat and protein. BMI, calculated as weight in kg divided by height in $\mathrm{m}^{2}$, was also considered. Trained researchers measured weight to the nearest $100 \mathrm{~g}$ using the Wedderburn Digital Chair Scale and height to the nearest $0 \cdot 1 \mathrm{~cm}$ using a Holtain stadiometer. Due to the narrow age range in the 14-year follow-up, age was not considered as a confounding factor.

\section{Statistical analyses}

Nutrient intakes, including GL measures, were adjusted for total energy using the residual method to control for confounding and reduce extraneous variation ${ }^{(26)}$. Continuous measures are expressed as means and standard deviations. Student's independent-sample $t$ tests, Mann-Whitney $U$ tests and $\chi^{2}$ tests were used to compare the characteristics of the included and excluded adolescent subjects. Logistic regression models were used to analyse the association between mealtime GL measures and the metabolic syndrome, adjusted for potential confounding variables and split by sex (due to significant interactions between sex and GL measures). Potential confounding variables were tested in the models. Nagelkerke $R^{2}$ values were compared between the models, with increasing values indicating a better $\mathrm{fit}^{(27)}$. Variables were retained as confounders in the model if they were significant or improved the fit of the model. Models were fitted with and without BMI to allow comparisons, because BMI is associated with the metabolic syndrome - the definition of the metabolic syndrome includes waist circumference. OR and 95\% CI were obtained for all variables. Where GL measures were found to be significant predictors of the metabolic syndrome, regression models were used to examine associations with continuous measures of metabolic syndrome components (waist circumference, blood pressure, and fasting serum TAG, fasting HDL-C and fasting plasma glucose concentrations). Components were logged as required to normalise data. BMI was included in each of these analyses, with the exception of waist circumference. No mathematical correction was made for multiple comparisons. Statistical analyses were performed using the Statistical Package for Social Sciences (SPSS Statistics for Windows, version 19.0; IBM Corporation), and significance level in the tests was set at 0.05.

\section{Results}

\section{Study population}

From the original cohort of 2868 at birth, 1286 adolescents who participated in the 14-year follow-up agreed to complete the $3 \mathrm{~d}$ food records. Adolescents who completed the $3 \mathrm{~d}$ food records were found to more likely have older mothers, a higher family income and a lower BMI compared with other adolescents in the follow-up who did not complete the $3 \mathrm{~d}$ food records ${ }^{(28)}$. Completed records were returned by 962 subjects $^{(3)}$. Of these, 822 records were considered to be complete and representative of usual diet. A total of five subjects were excluded as they had diagnosed diabetes, and none of the subjects was excluded for implausible energy intakes. A total of 516 non-diabetic adolescents provided food records where all the six meals had at least two GL values to average, and this 'two-meal valid' group was used in the statistical models. A comparison of the subject characteristics of the included ( $n$ 516) and excluded ( $n$ 306) groups from among the 822 adolescents with food records that were considered to be complete and representative of usual diet is given in Table 1. Daily dietary carbohydrate intake was found to be significantly higher in the excluded subject group $(P=0 \cdot 028)$.

\section{Mealtime glycaemic carbohydrate intake}

Meal GL values are given in Table 2. Dinner was the meal with the highest GL value (44.9 (SD 20.1)), followed by lunch (31.6 (SD 16.5)), breakfast (30.9 (SD 14.9)), afternoon tea (23.9 (SD 18.6)), morning tea (15.5 (SD 13.2)) and supper (10.7 (SD 11.5)). A breakdown of dietary intake and the prevalence of the metabolic syndrome by mean meal GL tertiles for boys and girls is also given in Table 2. Boys and girls with higher mean meal GL values were found to more likely have higher energy-adjusted carbohydrate intakes and lower protein and fat intakes when compared with boys and girls with lower mean meal GL values $(P<0.05)$. Among the 516 adolescents comprising the included group, 480 had data available to assess the metabolic syndrome, which was identified in seventeen (3.5\%) of the 480 subjects. Increasing risk of the metabolic syndrome with increasing mean meal GL tertiles was observed in boys but in not girls (Table 2).

\section{Associations with the metabolic syndrome}

The final logistic regression models included BMI, singleparent family, physical activity and daily protein intake as confounding variables. The other factors investigated did not contribute significantly to the fit of the models and so were not included as confounders. The results of the logistic regression analyses are given in Table 3; there was little difference in OR and significance when BMI was included or excluded as a confounder in these models. Daily GL was not a significant predictor of the metabolic syndrome. Breakfast GL was associated with an increased risk of the metabolic syndrome (OR 1.15, $95 \%$ CI 1.04, 1.27; $P<0 \cdot 01$ ) in girls. That is, for each unit increase in breakfast GL, the odds of developing the metabolic syndrome increased by a factor of 
Table 1. Comparison of the subject characteristics of the groups included in the study (minimum of two meals with a valid glycaemic load (GL)) and excluded from the study (due to $>20 \%$ dietary carbohydrate not assigned a glycaemic index, insufficient valid meal GL values, or diabetes) out of the adolescents who returned complete and representative food records ( $n$ 822)

(Mean values, standard deviations, number of participants and percentages)

\begin{tabular}{|c|c|c|c|c|c|}
\hline & \multicolumn{2}{|c|}{$\begin{array}{l}\text { Included group } \\
\quad(n 516)\end{array}$} & \multicolumn{2}{|c|}{$\begin{array}{l}\text { Excluded group } \\
\qquad(n 306)\end{array}$} & \multirow[b]{2}{*}{$P^{\star}$} \\
\hline & $n$ & $\%$ & $n$ & $\%$ & \\
\hline \multicolumn{6}{|l|}{ Subject characteristics } \\
\hline Sex (female) & 252 & $48 \cdot 8$ & 149 & $48 \cdot 7$ & 0.968 \\
\hline Weight categoriest & & & & & 0.053 \\
\hline Underweight & 30 & $5 \cdot 8$ & 25 & $8 \cdot 2$ & \\
\hline Normal weight & 352 & 68.5 & 225 & $73 \cdot 8$ & \\
\hline Overweight & 102 & 19.9 & 45 & $14 \cdot 7$ & \\
\hline Obese & 30 & $5 \cdot 8$ & 10 & $3 \cdot 3$ & \\
\hline Physical activity participation & & & & & 0.980 \\
\hline$\geq 4$ times/week & 179 & 34.8 & 108 & $35 \cdot 4$ & \\
\hline $1-3$ times/week & 288 & $56 \cdot 0$ & 170 & $55 \cdot 7$ & \\
\hline$\leq 1$ time/month & 47 & $9 \cdot 1$ & 27 & 8.8 & \\
\hline Screen time - computers, TV and video & & & & & 0.766 \\
\hline$\geq 4 \mathrm{~h} / \mathrm{d}$ & 159 & $31 \cdot 2$ & 94 & $31 \cdot 1$ & \\
\hline $2-4 \mathrm{~h} / \mathrm{d}$ & 201 & 39.5 & 126 & $41 \cdot 7$ & \\
\hline$<2 \mathrm{~h} / \mathrm{d}$ & 149 & $29 \cdot 3$ & 82 & $27 \cdot 2$ & \\
\hline Single-parent family & 97 & $19 \cdot 0$ & 47 & $15 \cdot 5$ & 0.210 \\
\hline Annual family income (pa, \$AUS) & & & & & 0.956 \\
\hline$<\$ 35000$ & 106 & 20.9 & 63 & 20.9 & \\
\hline$\$ 35001-\$ 70000$ & 180 & $35 \cdot 6$ & 110 & $36 \cdot 5$ & \\
\hline$>\$ 70001$ & 220 & 43.5 & 128 & $42 \cdot 5$ & \\
\hline Maternal education & & & & & 0.758 \\
\hline$<$ Year 12 & 240 & $46 \cdot 6$ & 146 & $47 \cdot 7$ & \\
\hline$\geq$ Year 12 & 275 & 53.4 & 160 & $52 \cdot 3$ & \\
\hline \multicolumn{6}{|l|}{ Dietary variables } \\
\hline Energy (kcal/d) & \multirow{2}{*}{\multicolumn{2}{|c|}{2225}} & & & 0.067 \\
\hline Mean & & & \multicolumn{2}{|c|}{2303} & \\
\hline SD & \multicolumn{2}{|c|}{579} & \multicolumn{2}{|c|}{584} & \\
\hline \multicolumn{6}{|l|}{ Energy $(\mathrm{kJ} / \mathrm{d})$} \\
\hline Mean & \multicolumn{2}{|c|}{9309} & \multicolumn{2}{|c|}{9636} & \\
\hline SD & \multicolumn{2}{|c|}{2423} & \multicolumn{2}{|c|}{2443} & \\
\hline Carbohydrate (g/d) & & & & & 0.028 \\
\hline Mean & \multicolumn{2}{|c|}{277} & \multicolumn{2}{|c|}{291} & \\
\hline SD & \multicolumn{2}{|c|}{79} & \multicolumn{2}{|c|}{87} & \\
\hline Protein (g/d) & & & & & 0.529 \\
\hline Mean & \multicolumn{2}{|c|}{$88 \cdot 4$} & \multicolumn{2}{|c|}{$89 \cdot 6$} & \\
\hline $\mathrm{SD}$ & \multicolumn{2}{|c|}{$26 \cdot 1$} & \multicolumn{2}{|c|}{$26 \cdot 5$} & \\
\hline Total fat $(\mathrm{g} / \mathrm{d})$ & & & & & 0.249 \\
\hline Mean & \multicolumn{2}{|c|}{$80 \cdot 7$} & \multicolumn{2}{|c|}{$82 \cdot 7$} & \\
\hline SD & & & & & \\
\hline Saturated fat $(\mathrm{g} / \mathrm{d})$ & & & & & 0.231 \\
\hline Mean & & & & & \\
\hline SD & & & & & \\
\hline
\end{tabular}

TV, television; pa, per annum.

${ }^{*}$ All comparisons of means for normally distributed scale variables were made using Student's independent-sample $t$ tests; MannWhitney $U$ tests were used where scale variables were not normally distributed. The $\chi^{2}$ test of contingencies was used to compare categorical variables of the two groups. $P<0.05$ (two-tailed) in all cases.

† Standard adolescent criteria were used to classify participants into BMI categories of underweight, normal weight, overweight and obese $^{(53,54)}$.

$1 \cdot 15$ (or equivalently by $15 \%$ ). With BMI being excluded from the model, breakfast GL was still a significant predictor (OR $1 \cdot 06,95 \%$ CI $1 \cdot 00,1 \cdot 12 ; P=0 \cdot 04)$. Breakfast GL was not a significant predictor of the metabolic syndrome in boys $(P=0 \cdot 15)$. No other GL values were significant predictors of the metabolic syndrome. When breakfast GL was examined in relation to each of the components of the metabolic syndrome in girls, it was found to be negatively associated with fasting HDL-C concentrations $(P=0.037 ; \beta=-0.004$, $95 \% \mathrm{CI}-0 \cdot 008,-0.002)$ and positively associated with fasting
TAG concentrations $(P=0.008 ; \beta=0.002$ for logged TAG values; $\exp (\beta)=1.002,95 \%$ CI $1.001,1.004)$. That is, for each unit increase in breakfast GL, there was a mean decrease in HDL-C concentrations of $0.004 \mathrm{mmol} / 1$ and a $0.2 \%$ increase in the geometric mean fasting TAG concentrations.

\section{Discussion}

The present study aimed to investigate the associations between mealtime measures of GL intake and metabolic 
Table 2. Glycaemic load (GL) variables and prevalence of the metabolic syndrome in Raine Study adolescents arranged according to tertiles of mean meal GL

(Mean values, standard deviations, number of participants and percentages)

\begin{tabular}{|c|c|c|c|c|c|c|c|c|c|c|c|c|c|c|}
\hline \multirow[b]{3}{*}{ Variables } & & & \multicolumn{6}{|c|}{ Boys } & \multicolumn{6}{|c|}{ Girls } \\
\hline & \multicolumn{2}{|c|}{$\begin{array}{l}\text { Whole group } \\
\quad(n 516)\end{array}$} & \multicolumn{2}{|c|}{$\begin{array}{l}\text { Low meal } \\
\text { GL† }(n 88)\end{array}$} & \multicolumn{2}{|c|}{$\begin{array}{l}\text { Medium meal } \\
\text { GL† ( } n \text { 82) }\end{array}$} & \multicolumn{2}{|c|}{$\begin{array}{l}\text { High meal } \\
\text { GL† ( } n \text { 94) }\end{array}$} & \multicolumn{2}{|c|}{$\begin{array}{l}\text { Low meal } \\
\text { GL† }(n 95)\end{array}$} & \multicolumn{2}{|c|}{$\begin{array}{l}\text { Medium meal } \\
\text { GL† ( } n \text { 94) }\end{array}$} & \multicolumn{2}{|c|}{$\begin{array}{l}\text { High meal } \\
\text { GL† }(n 78)\end{array}$} \\
\hline & Mean & SD & Mean & SD & Mean & SD & Mean & SD & Mean & SD & Mean & SD & Mean & SD \\
\hline \multicolumn{15}{|c|}{ Daily nutrient intakes $\ddagger$} \\
\hline \multicolumn{15}{|l|}{ Energy } \\
\hline kcal & 2225 & 579 & 2533 & 609 & 2430 & 637 & 2497 & 541 & 1892 & 378 & 1910 & 435 & $2057^{*}$ & 398 \\
\hline $\mathrm{kJ}$ & 9309 & 2423 & 10598 & 2548 & 10167 & 2665 & 10447 & 2264 & 7916 & 1582 & 7991 & 1820 & 8606 & 1665 \\
\hline Carbohydrate (g) & 277 & 79 & 249 & 28 & 277 & 20 & $304^{\star}$ & 22 & 253 & 20 & 278 & 17 & $303^{*}$ & 25 \\
\hline Protein $(\mathrm{g})$ & 88.4 & $26 \cdot 1$ & $97 \cdot 8$ & $17 \cdot 5$ & $91 \cdot 0$ & 13.9 & $84 \cdot 2^{*}$ & $14 \cdot 7$ & 93.9 & $11 \cdot 2$ & 85.4 & $11 \cdot 1$ & $78 \cdot 4^{*}$ & $12 \cdot 7$ \\
\hline Total fat (g) & $80 \cdot 7$ & $24 \cdot 7$ & $89 \cdot 3$ & $11 \cdot 2$ & 79.7 & 9.0 & $71 \cdot 2^{*}$ & 9.4 & 88.8 & 8.7 & 81.9 & 8.4 & $73.9^{*}$ & 9.8 \\
\hline Saturated fat (g) & 34.3 & $12 \cdot 3$ & 38.7 & $7 \cdot 7$ & 34.5 & $5 \cdot 8$ & $29 \cdot 6^{*}$ & $6 \cdot 0$ & $36 \cdot 8$ & 5.4 & $35 \cdot 1$ & $5 \cdot 7$ & $31 \cdot 2^{*}$ & $5 \cdot 8$ \\
\hline Daily GI (\%) & $54 \cdot 6$ & 4.9 & $51 \cdot 3$ & 4.1 & 55.0 & 3.5 & $57 \cdot 5^{\star}$ & 4.2 & 51.6 & 4.9 & $54 \cdot 3$ & 3.8 & $58 \cdot 1^{*}$ & 4.5 \\
\hline Daily GL & 152 & 45 & 126 & 16 & 152 & 10 & $175^{\star}$ & 15 & 131 & 13 & 150 & 9 & $175^{\star}$ & 16 \\
\hline \multicolumn{15}{|l|}{ GL variablesł } \\
\hline Breakfast GL & $30 \cdot 9$ & 14.9 & $26 \cdot 5$ & $11 \cdot 8$ & 34.6 & $14 \cdot 8$ & $35 \cdot 2^{*}$ & $16 \cdot 5$ & $25 \cdot 9$ & $9 \cdot 0$ & $30 \cdot 1$ & $10 \cdot 5$ & $32 \cdot 6^{*}$ & $12 \cdot 6$ \\
\hline Morning tea GL & $15 \cdot 5$ & $13 \cdot 2$ & $10 \cdot 7$ & 11.4 & 13.5 & $11 \cdot 1$ & $17 \cdot 6^{*}$ & $13 \cdot 7$ & 13.5 & 9.4 & $15 \cdot 8$ & $10 \cdot 6$ & $21 \cdot 9^{*}$ & $15 \cdot 2$ \\
\hline Lunch GL & 31.6 & $16 \cdot 5$ & $29 \cdot 8$ & $16 \cdot 8$ & 27.5 & 14.7 & $38.9^{*}$ & $15 \cdot 6$ & $26 \cdot 7$ & 11.8 & $32 \cdot 2$ & $12 \cdot 1$ & $33 \cdot 6^{*}$ & 14.4 \\
\hline Afternoon tea GL & 23.9 & $18 \cdot 6$ & $17 \cdot 2$ & $15 \cdot 1$ & $25 \cdot 3$ & $15 \cdot 9$ & $26 \cdot 6^{*}$ & $20 \cdot 8$ & $20 \cdot 0$ & $10 \cdot 6$ & $22 \cdot 6$ & 11.9 & $32 \cdot 1^{*}$ & $19 \cdot 4$ \\
\hline Dinner GL & 44.9 & $20 \cdot 1$ & $37 \cdot 3$ & $14 \cdot 0$ & $42 \cdot 6$ & $16 \cdot 2$ & $53 \cdot 9^{*}$ & $24 \cdot 0$ & $39 \cdot 1$ & $13 \cdot 2$ & $44 \cdot 1$ & $13 \cdot 9$ & $51 \cdot 8^{\star}$ & $18 \cdot 8$ \\
\hline Supper GL & $10 \cdot 7$ & 11.5 & $7 \cdot 7$ & 8.9 & $11 \cdot 8$ & $12 \cdot 8$ & $11 \cdot 8^{\star}$ & 14.7 & 9.0 & $6 \cdot 1$ & 11.4 & 8.4 & $12 \cdot 4^{*}$ & $9 \cdot 2$ \\
\hline Peak GL score & $42 \cdot 3$ & $15 \cdot 6$ & 38.5 & 14.5 & 41.7 & $15 \cdot 6$ & $50 \cdot 7^{*}$ & $19 \cdot 5$ & $36 \cdot 4$ & $10 \cdot 2$ & $40 \cdot 3$ & 1.6 & $46 \cdot 2^{*}$ & $16 \cdot 0$ \\
\hline \multicolumn{15}{|l|}{ Metabolic syndrome§ } \\
\hline \multicolumn{15}{|l|}{ Yes } \\
\hline$n$ & \multirow{2}{*}{\multicolumn{2}{|c|}{$\begin{array}{c}17 \\
3.5\end{array}$}} & \multicolumn{2}{|c|}{2} & \multicolumn{2}{|c|}{0} & \multicolumn{2}{|c|}{$6^{*}$} & \multicolumn{2}{|c|}{2} & \multicolumn{2}{|c|}{5} & \multicolumn{2}{|c|}{2} \\
\hline$\%$ & & & 2 & & 0 & & 6 & & & & & & & \\
\hline \multicolumn{15}{|l|}{ No } \\
\hline$n$ & \multicolumn{2}{|c|}{463} & \multicolumn{2}{|c|}{81} & \multicolumn{2}{|c|}{73} & \multicolumn{2}{|c|}{82} & \multicolumn{2}{|c|}{74} & & & & \\
\hline$\%$ & 96 & & 97 & & 10 & & 93 & & & & 9 & & & \\
\hline
\end{tabular}

GI, glycaemic index.

* Comparison between the highest and lowest tertiles; all comparisons of means for normally distributed scale variables were made using Student's independent-sample $t$ tests; Mann-Whitney $U$ tests were used where scale variables were not normally distributed. The $\chi^{2}$ test of contingencies was used to compare categorical variables of the two populations. $P<0.05$ (two-tailed) in all cases.

† Arranged into tertiles of mean meal GL, where mean meal $\mathrm{GL}=\Sigma$ (breakfast $\mathrm{GL}+$ morning tea $\mathrm{GL}+$ lunch $\mathrm{GL}+$ afternoon tea $\mathrm{GL}+$ dinner $\mathrm{GL}+$ supper $\mathrm{GL}$ ) $/ 6$.

$\ddagger$ Daily intakes adjusted for energy.

$\S$ The International Diabetes Foundation definition of the metabolic syndrome, i.e. the presence of a high waist circumference in addition to two or more of the following: high systolic or diastolic blood pressure; high fasting serum TAG concentrations; low serum HDL-cholesterol concentrations; high plasma glucose concentrations; cut points for the categorisation of these high and low subgroups vary by sex and age, as has been published previously ${ }^{(22)}$.

syndrome risk as well as metabolic syndrome components in 14-year-old adolescents. It was hypothesised that meal-based GL values would be better predictors of metabolic syndrome risk than a daily GL value. In the 516 adolescents comprising the included group, no significant association was found between daily GL values and metabolic syndrome risk. However, breakfast GL was found to be a significant independent predictor of the metabolic syndrome in the same group. As GL values were being compared on a meal basis, adolescents were excluded where it was not possible to accurately and consistently assign meal GL values. In a previously published study of the larger Raine Study cohort, a significant association was found between daily GL and the metabolic syndrome ${ }^{(3)}$. It is likely that a reduced sample size meant that we could no longer detect a significant association with daily GL. We expect a low prevalence in a paediatric population cohort rather than in a clinical group, and caution must be exercised when interpreting the results due to low statistical power to find associations with dietary components ${ }^{(29)}$. However, our findings suggest that breakfast GL may be a more sensitive predictor than a daily GL value in the study group.
Breakfast GL was found to be significantly associated with the odds of developing the metabolic syndrome in girls, but not in boys. This association was seen independently and dependently of BMI and so BMI does not appear to mediate the observed association. To put this association into perspective, the results of the present study suggest that if an additional slice of white bread $(G L=12)$ were added on top of the girls' existing breakfast, the theoretical associated odds of developing the metabolic syndrome would be 5.35 times greater, with an associated 95\% CI of 1.60-17.6 times. It must be noted that the CI obtained in the present study was large, due in part to the low prevalence of the metabolic syndrome in the study group (seventeen adolescents; nine girls). Breakfast GL was also found to be significantly associated with two components of the metabolic syndrome: decreased fasting HDL-C concentrations and increased fasting TAG concentrations.

Almost all previous studies using daily GI/GL values have not been able to distinguish between different mealtime effects on glucose and insulin responses, and this may have contributed to conflicting results on whether dietary glycaemic carbohydrate intake is a useful predictor of chronic disease 
Table 3. Meal, peak score and daily glycaemic load $(\mathrm{GL})^{\star}$ variables and risk of the metabolic syndromet in Raine Study adolescents ( $n$ 516) in unadjusted and adjusted logistic regression models (with and without BMI)‡ (Odds ratios and $95 \%$ confidence intervals)

\begin{tabular}{|c|c|c|c|c|c|c|}
\hline \multirow[b]{2}{*}{ Meal GL variables (BMI excluded/included)‡ } & \multicolumn{3}{|c|}{ Girls (n 252) } & \multicolumn{3}{|c|}{ Boys ( $n$ 264) } \\
\hline & OR & $95 \% \mathrm{Cl}$ & $P$ & OR & $95 \% \mathrm{Cl}$ & $P$ \\
\hline \multicolumn{7}{|l|}{ Breakfast GL } \\
\hline Unadjusted & 1.05 & $0.99,1.11$ & 0.07 & 1.01 & $0.97,1.06$ & 0.51 \\
\hline Adjusted, BMI excluded & 1.06 & $1.00,1 \cdot 12$ & 0.04 & 1.04 & $0.98,1.09$ & 0.18 \\
\hline Adjusted, BMI included & $1 \cdot 15$ & $1.04,1.27$ & $<0.01$ & 0.83 & $0.64,1.07$ & 0.15 \\
\hline \multicolumn{7}{|l|}{ Lunch GL } \\
\hline Unadjusted & 1.04 & $0.99,1.09$ & 0.06 & 1.03 & $0.99,1.07$ & 0.09 \\
\hline Adjusted, BMI excluded & 1.04 & $0.99,1.08$ & 0.15 & 1.04 & $1.00,1.09$ & 0.06 \\
\hline Adjusted, BMI included & 1.04 & $0.99,1 \cdot 10$ & 0.14 & 1.05 & $0.97,1.15$ & 0.24 \\
\hline \multicolumn{7}{|l|}{ Dinner GL } \\
\hline Unadjusted & 1.00 & $0.95,1.04$ & 0.84 & 0.99 & $0.95,1.03$ & 0.56 \\
\hline Adjusted, BMI excluded & 0.98 & $0.94,1.03$ & 0.44 & 0.97 & $0.93,1.01$ & $0 \cdot 14$ \\
\hline Adjusted, BMI included & 0.97 & $0.91,1.04$ & 0.43 & 0.96 & $0.89,1.02$ & 0.19 \\
\hline \multicolumn{7}{|l|}{ Peak GL score } \\
\hline Unadjusted & 1.01 & $0.97,1.07$ & 0.58 & 1.01 & $0.97,1.05$ & 0.70 \\
\hline Adjusted, BMI excluded & 1.00 & $0.94,1.05$ & 0.94 & 0.99 & $0.95,1.04$ & 0.78 \\
\hline Adjusted, BMI included & 1.01 & $0.95,1.08$ & 0.71 & 0.95 & $0.86,1.04$ & 0.24 \\
\hline \multicolumn{7}{|l|}{ Daily GL } \\
\hline Unadjusted & 1.00 & $0.98,1.02$ & 0.77 & 1.01 & $0.99,1.02$ & 0.48 \\
\hline Adjusted, BMI excluded & 1.00 & $0.98,1.02$ & 0.90 & 1.00 & $0.99,1.02$ & 0.64 \\
\hline Adjusted, BMI included & 1.01 & $0.99,1.04$ & 0.44 & 1.03 & $0.99,1.06$ & 0.19 \\
\hline
\end{tabular}

${ }^{*}$ All GL variables were adjusted for energy.

†The age-specific International Diabetes Foundation definition of the metabolic syndrome was used ${ }^{(22)}$

‡ Logistic regression models were adjusted for single-parent family, physical activity and energy-adjusted daily protein intake, with BMI excluded or included as an additional confounder.

$\operatorname{risk}^{(9,11-14,30-34)}$. Our findings suggest that breakfast GL may be particularly important. Blood glucose and insulin responses have been shown to be proportional to breakfast GL in clinical trials $^{(31,32)}$. Bao et al. ${ }^{(31)}$ suggest that breakfast metabolic responses may not necessarily reflect responses to other meals. Clinical trials carried out in adolescents have shown the benefits of consuming low-GI carbohydrate at breakfast ${ }^{(35)}$, with increased satiety and reduced consumption at an ad libitum lunch, while breakfasts with sufficiently low-GI, multi-grain cereals may produce second meal effects that can last through to lunch or beyond ${ }^{(36)}$. It is possible that a low-GL breakfast may have the benefit of decreasing the amount eaten at lunch (and potentially the lunch GL), thus reducing metabolic syndrome risk associated with both meals. Effects may differ by age - O'Sullivan et al. ${ }^{(16)}$ showed that increasing lunch GL is significantly associated with an increased risk of insulin resistance, along with the peak GL score, in older women.

In the present study, two components of the metabolic syndrome, decreased fasting HDL-C concentrations and increased fasting TAG concentrations, were found to be significantly associated with increasing breakfast GL. Other studies carried out in both youth and adults have also found similar associations with GL. In a randomised controlled trial involving thirty-two healthy 11-25-year-olds, higher-GL diets were found to be associated with lower HDL-C concentrations ${ }^{(37)}$. A systematic review and meta-analysis ${ }^{(10)}$ has concluded that lower-GL diets are associated with lower fasting plasma TAG concentrations in adults. In an adult male population, fasting TAG concentrations were found to increase with increasing dietary GI, but not with GL, and HDL-C concentrations were found to decrease with increasing $\mathrm{GL}^{(38)}$. The risk of developing the metabolic syndrome was found to be associated with daily GI and GL in Korean women (but not in men), with high TAG concentrations and low HDL-C concentrations, components that are associated with high intakes. Although more research is needed to expand on our findings, there are potential mechanisms to explain the results of the present study. Habitual intake of high-GL meals can result in hyperglycaemia, hyperinsulinaemia and disturbed lipid metabolism ${ }^{(7)}$, which have been reported to be associated with the development of chronic diseases such as the metabolic syndrome and consequent type 2 diabetes and heart disease ${ }^{(31,39-41)}$. Following a high peak in glucose concentrations and subsequently insulin concentrations, postprandial hypoglycaemia is common 4-6h after the consumption of a high-GL meal. This can stimulate the secretions of counter-regulatory hormones that raise glucose and NEFA concentrations ${ }^{(7)}$. This is associated with increased concentrations of inflammatory mediators and TAG and decreased concentrations of HDL-C $\mathrm{C}^{(42)}$.

In the present study, significant associations were found in girls, but not in boys. Higher-GL diets have previously been reported to be associated with a greater risk of the metabolic syndrome in women, but not in men ${ }^{(43)}$. Females may be more innately insulin-resistant than males due to specific sex-linked gene expression, leading to changes in receptor and signalling pathways ${ }^{(44)}$. In puberty, there is a natural tendency for girls to exhibit more fat gain relative to boys ${ }^{(44,45)}$. Hormones in girls such as oestradiol favour fat deposition, 
while those in boys favour muscle tissue accumulation ${ }^{(45)}$. Increased oestradiol concentrations are associated with increased subcutaneous fat deposition and insulin responses and decreased fatty acid oxidation ${ }^{(46)}$. Higher fat stores and insulin concentrations in turn increase the secretion of leptin; increased leptin concentrations lead to increased oestradiol concentrations and subsequently insulin-like growth factor 1 concentrations, further increasing insulin secretion and fat storage ${ }^{(45)}$. Although highly speculative, the effect of hormonal surges at a key stage in puberty is a possible reason for an increased sensitivity to GL in relation to metabolic syndrome risk during this time period.

Daily dietary protein intake was found to be an important confounding factor in the association of breakfast GL with metabolic syndrome risk in girls. In the present study, the adolescents were found to consume breakfasts with a relatively high GL, but low protein content, when compared with lunch and dinner. Increasing protein consumption at meals lowers the glycaemic response by delaying gastric emptying ${ }^{(47)}$. A high-protein, low-GI diet was found to produce a combined beneficial effect attributed to reduced insulin responses, increased satiety and decreased energy intake in children (5-18 years) in the Diet, Obesity and Genes (DiOGenes) dietary study ${ }^{(48)}$, while higher $v$. lower protein intake was found to be associated with lower waist circumference and lower LDL-cholesterol concentrations in another paediatric subset of this study ${ }^{(49)}$. Higher-protein breakfasts may have the ability to attenuate high-GL responses sufficiently to reduce metabolic syndrome risk. Consumption of quality protein at breakfast may lower the meal GL by promoting satiety and by displacing carbohydrate. Further research is required to test this concept.

\section{Strengths and limitations}

The strengths of the present study include the use of $3 \mathrm{~d}$ food records, which enabled investigation of GL at a mealtime level. It also allowed for carrying out sex-specific analysis of the study group. The limitations include the inability to generalise the results to other Western adolescent populations, with the adolescents completing food records in the present study being found to more likely have a lower BMI and older mothers and come from households with a higher annual income. Among the 480 adolescents with complete meal GL data, seventeen were found to have diagnosed metabolic syndrome, of whom nine were females. Subjects who were excluded had significantly higher intakes of carbohydrate (Table 1), and this may have meant that some associations with higher intakes went undetected. The bulk of published GI values come from Australia and the USA ${ }^{(50)}$, and despite the high representation of Australian foods, there is a need for a larger GI database of carbohydrate foods commonly consumed by younger populations, such as fast foods and snack bars. Consumption of foods that did not have GI values often occurred at the same mealtime on two consecutive days, which effectively led to the exclusion of a subject from the study each time (via the previously determined exclusion criterion requiring at least two GL values to average for any one meal). Although many adolescents were excluded due to the strict criteria implemented in the present study, this method helped to maintain accuracy of the data by ensuring that the meal GL values represented a true reflection of the foods reported. Although attempts were made to minimise under- and over-reporting through the use of cut-offs previously used in studies carried out in adolescents, this method is imprecise and it is possible that adolescents who were misreporting their intake were included in the study. It has been suggested that adolescents with a higher BMI (and therefore at a higher risk of the metabolic syndrome) are more likely to misreport dietary intake ${ }^{(18)}$, and this could affect the associations observed. In addition, the present study is a cross-sectional snapshot of the prospective cohort, and as such causality cannot be established.

\section{Implications}

In the present study, we hypothesised that meal-based GL values would be better predictors of metabolic syndrome risk than a daily GL value; breakfast GL did appear to have a more sensitive association. Adolescence is an important time period for establishing dietary patterns into adulthood, and insights into their impact on disease processes may provide meaningful data to formulate dietary advice. Although causality cannot be determined from the present study, it is possible that the inclusion of low-GL foods to breakfast may be beneficial for girls. Our findings support previous recommendations made in this regard around consumption of a low-GL breakfast ${ }^{(51,52)}$.

\section{Acknowledgements}

The core management funding for the Raine Study was provided by the University of Western Australia (UWA), the Raine Medical Research Foundation at the UWA, the Faculty of Medicine, Dentistry and Health Sciences at the UWA, the Telethon Institute for Child Health Research, the Women and Infants Research Foundation, the Telethon Institute for Child Health Research and Curtin University. The 14-year followup study received funding from the NH\&MRC (Sly et al., ID 211912), a NH\&MRC Program Grant (Stanley et al., ID 003209), Healthway WA (Beilin et al.), the National Heart Foundation, Beyond Blue and the Raine Foundation.

The authors thank the Raine Study participants and their families who took part in the study and all the members of the Raine Study team for their expertise in cohort coordination and data collection. They also thank Timothy du Heaume and Kester Nicholl of Curtin University for technical support with data entry.

The authors' contributions are as follows: W. H. O., L. J. B. and T. A. M. were involved in data collection, T. A. O. conceptualised and supervised the research, A. N., M. d. H., A. P. B. and T. A. O. were involved in data analysis and manuscript drafting. All authors were involved in the review of the manuscript. 


\section{References}

1. Cook S, Weitzman M, Auinger P, et al. (2003) Prevalence of a metabolic syndrome phenotype in adolescents: findings from the third National Health and Nutrition Examination Survey, 1988-1994. Arch Pediatr Adolesc Med 157, 821-827.

2. Lin H-J, Lee B-C, Ho Y-L, et al. (2009) Postprandial glucose improves the risk prediction of cardiovascular death beyond the metabolic syndrome in the nondiabetic population. Diabetes Care 32, 1721-1726.

3. O'Sullivan TA, Lyons-Wall P, Bremner AP, et al. (2010) Dietary glycaemic carbohydrate in relation to the metabolic syndrome in adolescents: comparison of different metabolic syndrome definitions. Diabet Med 27, 770-778.

4. Cameron AJ, Magliano DJ, Zimmet PZ, et al. (2007) The metabolic syndrome in Australia: prevalence using four definitions. Diabetes Res Clin Pract 77, 471-478.

5. Jenkins DJ, Wolever TM, Taylor RH, et al. (1981) Glycemic index of foods: a physiological basis for carbohydrate exchange. Am J Clin Nutr 34, 362-366.

6. Esfahani A, Wong JMW, Mirrahimi A, et al. (2009) The glycemic index: physiological significance. J Am Coll Nutr 28, Suppl., 439S-445S.

7. Ludwig DS (2002) The glycemic index: physiological mechanisms relating to obesity, diabetes, and cardiovascular disease. JAMA 287, 2414-2423.

8. Brand-Miller J, McMillan-Price J, Steinbeck K, et al. (2008) Carbohydrates - the good, the bad and the whole grain. Asia Pac J Clin Nutr 17, Suppl. 1, 16-19.

9. Du H, van der ADL, van Bakel MME, et al. (2008) Glycemic index and glycemic load in relation to food and nutrient intake and metabolic risk factors in a Dutch population. Am J Clin Nutr 87, 655-661.

10. Livesey G, Taylor R, Hulshof $\mathrm{T}$, et al. (2008) Glycemic response and health - a systematic review and metaanalysis: relations between dietary glycemic properties and health outcomes. Am J Clin Nutr 87, 258S-268S.

11. Barclay AW, Petocz P, McMillan-Price J, et al. (2008) Glycemic index, glycemic load, and chronic disease risk a meta-analysis of observational studies. Am J Clin Nutr $\mathbf{8 7}, 627-637$.

12. McKeown NM, Meigs JB, Liu S, et al. (2004) Carbohydrate nutrition, insulin resistance, and the prevalence of the metabolic syndrome in the Framingham Offspring Cohort. Diabetes Care 27, 538-546.

13. Mayer-Davis EJ, Dhawan A, Liese AD, et al. (2006) Towards understanding of glycaemic index and glycaemic load in habitual diet: associations with measures of glycaemia in the Insulin Resistance Atherosclerosis Study. Br J Nutr 95, 397-405.

14. Sahyoun NR, Anderson AL, Tylavsky FA, et al. (2008) Dietary glycemic index and glycemic load and the risk of type 2 diabetes in older adults. Am J Clin Nutr 87, 126-131.

15. Hui LL \& Nelson EAS (2006) Meal glycaemic load of normalweight and overweight Hong Kong children. Eur J Clin Nutr 60, 220-227.

16. O'Sullivan TA, Bremner AP, O'Neill S, et al. (2010) Comparison of multiple and novel measures of dietary glycemic carbohydrate with insulin resistant status in older women. Nutr Metab 7, 25-34.

17. Newnham JP, Evans SF, Michael CA, et al. (1993) Effects of frequent ultrasound during pregnancy: a randomised controlled trial. Lancet 342, 887-891.

18. Rockett HRH, Breitenbach M, Frazier AL, et al. (1997) Validation of a youth/adolescent food frequency questionnaire. Prev Med 26, 808-816.
19. Ambrosini GL, de Klerk NH, O'Sullivan TA, et al. (2009) The reliability of a food frequency questionnaire for use among adolescents. Eur J Clin Nutr 63, 1251-1259.

20. Candilo KDI, Oddy W, Miller M, et al. (2007) Follow-up phone calls increase nutrient intake estimated by three-day food diaries in 13-year-old participants of the Raine study. Nutr Diet 64, 165-171.

21. University of Sydney (2008) Home of the Glycemic Index - GI Database. http://www.glycemicindex.com/ (accessed 2007-2008)

22. Jolliffe CJ \& Janssen I (2007) Development of age-specific adolescent metabolic syndrome criteria that are linked to the Adult Treatment Panel III and International Diabetes Federation criteria. J Am Coll Cardiol 49, 891-898.

23. Steinberger J, Daniels SR, Eckel RH, et al. (2009) Progress and challenges in metabolic syndrome in children and adolescents: a scientific statement from the American Heart Association Atherosclerosis, Hypertension, and Obesity in the Young Committee of the Council on Cardiovascular Disease in the Young; Council on Cardiovascular Nursing; and Council on Nutrition, Physical Activity, and Metabolism. Circulation 119, 628-647.

24. Tanner J (1962) Growth at Adolescence: With a General Consideration of the Effects of Hereditary and Environmental Factors Upon Growth and Maturation from Birth to Maturity. Oxford: Blackwell Scientific.

25. Duke PM, Litt IF \& Gross RT (1980) Adolescents' selfassessment of sexual maturation. Pediatrics 66, 918-920.

26. Willett WC, Howe GR \& Kushi LH (1997) Adjustment for total energy intake in epidemiologic studies. Am J Clin Nutr $\mathbf{6 5}$, Suppl. 4, 1220S-1228S.

27. Nagelkerke NJD (1991) A note on a general definition of the coefficient of determination. Biometrika 78, 691-692.

28. O'Sullivan TA, Ambrosini GL, Beilin LJ, et al. (2011) Dietary intake and food sources of fatty acids in Australian adolescents. Nutrition 27, 153-159.

29. Eisenmann J (2008) On the use of a continuous metabolic syndrome score in pediatric research. Cardiovasc Diabetol $7,17$.

30. Finley CE, Barlow CE, Halton TL, et al. (2010) Glycemic index, glycemic load, and prevalence of the metabolic syndrome in the cooper center longitudinal study. $J \mathrm{Am}$ Diet Assoc 110, 1820-1829.

31. Bao J, Atkinson F, Petocz P, et al. (2011) Prediction of postprandial glycemia and insulinemia in lean, young, healthy adults: glycemic load compared with carbohydrate content alone. Am J Clin Nutr 93, 984-996.

32. Galgani J, Aguirre C \& Díaz E (2006) Acute effect of meal glycemic index and glycemic load on blood glucose and insulin responses in humans. Nutr J 5, 22.

33. Sluijs I, van der Schouw YT, van der A DL, et al. (2010) Carbohydrate quantity and quality and risk of type 2 diabetes in the European Prospective Investigation into Cancer and Nutrition-Netherlands (EPIC-NL) study. Am J Clin Nutr 92, 905-911.

34. Vrolix R \& Mensink RP (2010) Effects of glycemic load on metabolic risk markers in subjects at increased risk of developing metabolic syndrome. Am J Clin Nutr 92, 366-374.

35. Warren JM, Henry CJ \& Simonite V (2003) Low glycemic index breakfasts and reduced food intake in preadolescent children. Pediatrics 112, e414.

36. Nilsson AC, Östman EM, Granfeldt Y, et al. (2008) Effect of cereal test breakfasts differing in glycemic index and content of indigestible carbohydrates on daylong glucose tolerance in healthy subjects. Am J Clin Nutr 87, 645-654. 
37. Slyper A, Jurva J, Pleuss J, et al. (2005) Influence of glycemic load on HDL cholesterol in youth. Am J Clin Nutr 81, 376-379.

38. Mosdøl A, Witte DR, Frost G, et al. (2007) Dietary glycemic index and glycemic load are associated with high-densitylipoprotein cholesterol at baseline but not with increased risk of diabetes in the Whitehall II study. Am J Clin Nutr 86, $988-994$

39. McMillan-Price J, Petocz P, Atkinson F, et al. (2006) Comparison of 4 diets of varying glycemic load on weight loss and cardiovascular risk reduction in overweight and obese young adults: a randomized controlled trial. Arch Intern Med 166, 1466-1475.

40. Sieri S, Krogh V, Berrino F, et al. (2010) Dietary glycemic load and index and risk of coronary heart disease in a large Italian cohort: the EPICOR study. Arch Intern Med 170, 640-647.

41. Berkey C, Rockett H, Gillman M, et al. (2003) Longitudinal study of skipping breakfast and weight change in adolescents. Int J Obes 27, 1258-1266.

42. Levitan EB, Cook NR, Stampfer MJ, et al. (2008) Dietary glycemic index, dietary glycemic load, blood lipids, and C-reactive protein. Metabolism 57, 437-443.

43. Kim K, Yun SH, Choi BY, et al. (2008) Cross-sectional relationship between dietary carbohydrate, glycaemic index, glycaemic load and risk of the metabolic syndrome in a Korean population. Br J Nutr 100, 576-584.

44. Mittendorfer B (2005) Insulin resistance: sex matters. Curr Opin Clin Nutr Metab Care 8, 367-372.

45. Casazza K \& Thomas O (2009) Do dietary modifications made prior to pubertal maturation have the potential to decrease obesity later in life? A developmental perspective. Infant Child Adolesc Nutr 1, 271-281.

46. Power ML \& Schulkin J (2008) Sex differences in fat storage, fat metabolism, and the health risks from obesity: possible evolutionary origins. Br J Nutr 99, 931-940.

47. Ludwig DS \& Ebbeling CB (2010) Weight-loss maintenance mind over matter? $N$ Engl J Med 363, 2159-2161.

48. Papadaki A, Linardakis M, Larsen TM, et al. (2010) The effect of protein and glycemic index on children's body composition: the DiOGenes randomized study. Pediatrics 126, 997-998.

49. Damsgaard CT, Papadaki A, Jensen SM, et al. (2013) Higher protein diets consumed ad libitum improve cardiovascular risk markers in children of overweight parents from eight European countries. J Nutr 143, 810-817.

50. Aston LM, Jackson D, Monsheimer S, et al. (2010) Developing a methodology for assigning glycaemic index values to foods consumed across Europe. Obes Rev 11, 92-100.

51. Kamada I, Truman L, Bold J, et al. (2011) The impact of breakfast in metabolic and digestive health. Gastroenterol Hepatol Bed Bench 4, 76-85.

52. Agostoni C \& Brighenti F (2010) Dietary choices for breakfast in children and adolescents. Crit Rev Food Sci Nutr 50 , $120-128$

53. Cole TJ, Flegal KM, Nicholls D, et al. (2007) Body mass index cut offs to define thinness in children and adolescents: international survey. BMJ 335, 194-201.

54. Cole TJ, Bellizzi MC, Flegal KM, et al. (2000) Establishing a standard definition for child overweight and obesity worldwide: international survey. BMJ 320, 1240-1243. 